\title{
Measurement of Longitudinal Spherical Aberration in the Extra-Axial Region of Lenses
}

\author{
Francis E. Washer and Walter R. Darling
}

\begin{abstract}
(April 10, 1962)
A method of measuring longitudinal spherical and chromatic aberration in the extraaxial region of lenses is described. The method employs an especially constructed optical T-bench equipped with nodal slide and angle-measuring telescope. The determinations are based upon measurements of angular deviations in selected small regions of the collimated beam emergent from the lens under test. The underlying theory of the method is presented together with a brief description of the apparatus used and techinque of measurement. Results of measurement on three lenses are included.
\end{abstract}

\section{Introduction}

Longitudinal spherical aberration is one of the prime causes that prevent a given lens from yielding optimum imagery. The measurement of this aberration is therefore of some interest, as the probable image forming qualities may be inferred from an analysis of the results of such measurements. Such measurements are also of interest in a diagnostic sense in that the results of measurement serve to explain why the performance of a given lens may fall short of expectations. In an earlier paper ${ }^{i}$ the senior author described a simple, rapid method of measuring the axial longitudiaal spherical aberration in an indirect manner. In this process, the lens is used to collimate the light from a point source located in the focal plane and the angular deviations of adjacent portions of the emergent beam from parallelism are measured; the longitudinal spherical aberration is then determined by computations using the angular displacements.

The present paper extends this process to the extra-axial region. The problem is somewhat more complicated in the extra-axial region, as the lens is not rotationally symmetric about a chief ray inclined at angle $\beta$ to the axis; so adequate information can be gained only by measuring in two meridians. The measurement of longitudinal chromatic aberration can readily be performed by the same process. Results of measurement are presented on three lenses.

\section{Theory of the Method}

The manner in which the values of longitudinal spherical aberration are derived from measurements of angular deviations from parallelism in a collimated beam of light emergent from a lens under test is described in detail in an earlier paper (see footnote 1). In this earlier paper, the discussion was confined to the situation where the luminous object was located on the optical axis at or near the focus and measurements were made in the axial

${ }_{1}^{1}$ F. E. Washer, Optical T-bench method of measuring longitudinal spherical aberration. J. Research NBS 61, 31 (1958) RP2880. collimated beam. Under these conditions, shown schematically in figure $1 \mathrm{a}$, the relation governing $\Delta f$, the displacement $00^{\prime}$ in the figure and $\epsilon$, the change in angular deviation of a small portion of the collimated beam located at height $h$ from the optical axis was shown to be

$$
\Delta f=\frac{\epsilon f^{2}}{h}
$$

where $f$ is the equivalent focal length of the lens under test.

For an ideal lens, the quantity $\epsilon / h$ is invariant with varying $h$. For a lens affected with longitudinal spherical aberration, the quantity $\epsilon / h$ varies with $h$, and measurement of $\epsilon$ as a function of $h$ permits the evaluation of longitudinal spherical abberration.
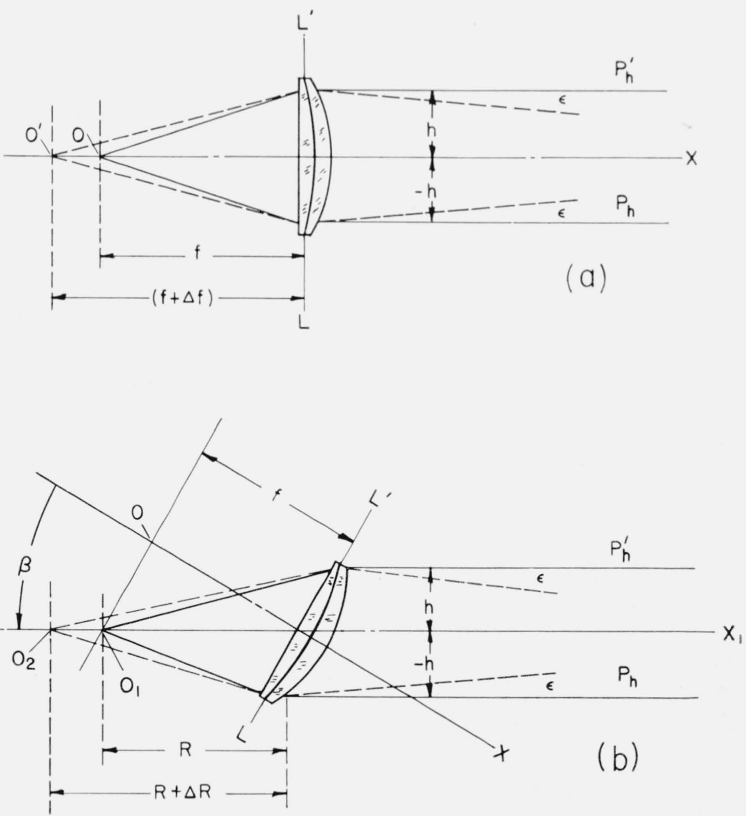

Figure 1. Schematic drawing of a lens showing (a) arrangement for measurement of longitudinal spherical aberration on axis and $(b)$ arrangement for measurement of longitudinal spherical aberration along a chief ray inclined at angle $\beta$ to the optical axis. 
In the present study, the chief interest is in the magnitude of the longitudinal spherical aberration as measured along a chief ray inclined at angle $\beta$ to the optical axis of the lens system. It is clear from figure $1 \mathrm{~b}$, that the same principles are involved as on the axis. Consequently the following modification of eq (1),

$$
\Delta R=\frac{\epsilon R^{2}}{h}
$$

is satisfactory in determining the displacement $0_{1} 0_{2}$ of the focus along the chief ray with zone height $h$. In the above equation,

$$
R=f \sec \beta,
$$

$h$ is measured normal to the chief ray, and $\Delta R=0_{1} 0_{2}$ is measured along the chief ray.

\section{Method of Measurement}

\subsection{In the Tangential Fan}

The method of measurement is essentially the same as that for determination of axial longitudinal spherical aberration. However, some changes in procedure are necessary to make measurements along the chief ray inclined at angle $\beta$ to the axis. For measurements in the tangential fan, the apparatus is arranged as shown in figure 2. Initially the lens is placed on the nodal slide with its front facing the pentaprism through which the angular deviations are viewed. The lens is adjusted until its rear nodal point is in the vertical axis of rotation of the nodal slide and the illuminated target reticle is located at the rear focal point of the lens. In

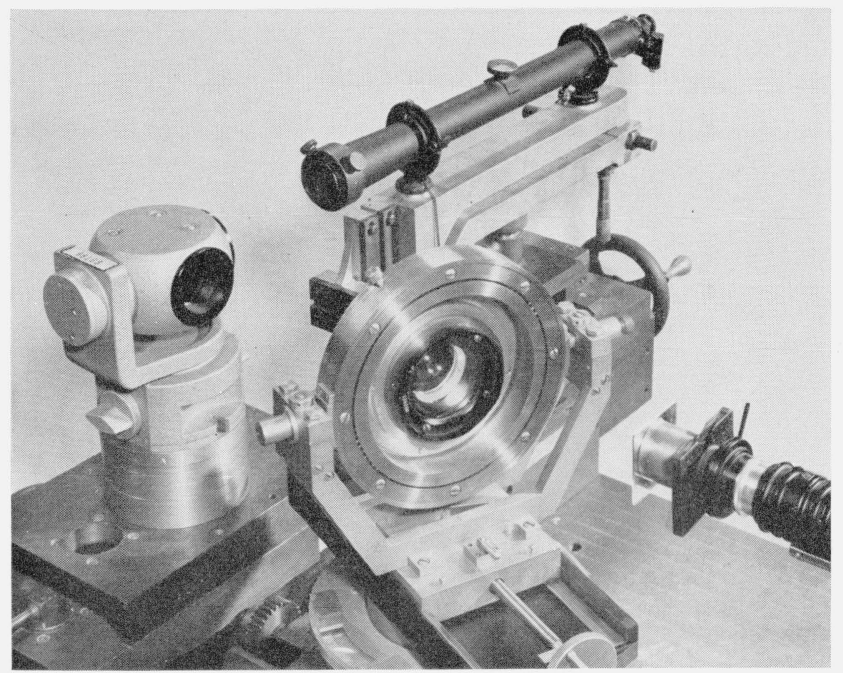

FIGURE 2. Test equipment arranged for measurements in the tangential fan.

The lens under test is shown mounted on the nodal slide in the center of the photograph; the lens has been rotated through an angle $\boldsymbol{\beta}$ about the vertical axis. The target reticle and illuminating system are shown at the right with axis. The target reticle and illuminating system are shown at the right with the target reticle facing the rear of the lens. The parallel beam of light emergent
from the front of the lens is turned through $90^{\circ} \mathrm{by}$ the pentaprism in the left of the scene into the viewing telescope at the rear. The diaphragm covering the telescope objective limits the beam to a small pencil of rays. addition, the optical axis of the lens is set parallel to the bench ways upon which the lens and target supports are located. When the target is illuminated, the light proceeding from the target falls upon the rear surface of the lens and emerges from the front as a collimated beam of light. This collimated beam is incident upon one face of the pentaprism mounted on the ways of the second bench and is directed into the viewing telescope located as shown in figure 2 .

The telescope objective is equipped with a diaphragm having a small central aperture which is centered with respect to the telescope objective. When an observer looks through the telescope, he sees the image of the illuminated target (either a cross or pinhole pattern) superimposed on the crosshairs in the ocular of the viewing telescope. When the slide carrying the pentaprism is moved along the bench ways, the observer sees the target through successive small areas of the lens along its diameter. In the event of movement of the image, it can be brought into coincidence with the crosshairs by appropriate movement of the transverse micrometer. Movement of the transverse micrometer changes the direction of pointing of the viewing telescope. The lateral movement of the micrometer is directly proportional to the angular change in pointing. Hence the change in pointing is a measure of the change in direction of a small circular beam of light emergent from a given area of the lens under test from the direction of another similar area lying along the same diameter of the lens. This angular deviation $\epsilon$ is measured in scale divisions read on the transverse micrometer. The value of $\epsilon$ in scale divisions may be converted to radians on multiplying by the calibration constant of the micrometer. For comparison purposes the value of $\epsilon$ in scale divisions may be used directly, but is usually converted to radians for the final evaluation of the longitudinal spherical aberration.

It is customary to select a size of diaphragm opening small in comparison with the area of the lens under test and to select a series of steps so that measurements of the deviation of these successive small emergent beams can be made for an entire diameter of the lens. If the lens under test has been properly focused, the angular deviations noted arise from parallax (or angular spherical aberration) of the lens under test.

The scale on the bench carrying the pentaprism is used in making the successive settings at selected intervals. These settings are recorded and the difference of a given bench scale reading from the one in the central position is the zone height, $h$. The readings of the angular micrometer are taken for each value of $h$ and the difference of a given micrometer reading from that one in the central position is recorded as $\epsilon$.

The longitudinal spherical aberration usually varies with the wavelength of the image forming light. It is therefore necessary when making measurements to know the wavelength of the light used. While it would be desirable to use monochromatic light, useful measurements can be obtained 
TABLE 1. Filters used in controlling illumination of target reticle*

Filter Nos, 58 and 25 transmit a moderately wide band of wavelengths; filter No. 73 is a narrow band filter. The column headed dominant wavelength gives the value of the wavelength that appears to be dominant in the light transmitted by the filter when illuminated by standard illuminant " $C$ ".

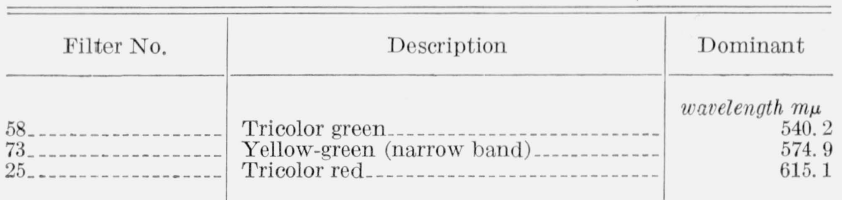

*Handbook of Chemistry and Physies (37th Ed.) pp. 2730-2734, Chemical Rubber Publ. Co., Cleveland, Ohio (1955-56).

using filters to control the quality of the light. Filters of the type used in photographic processes are suitable for the purpose. Moreover, results obtained with these filters present a picture of the manner in which longitudinal spherical aberration is operative under conditions of use. In the present study, three Wratten light filters were used; their characteristics are given in table 1. Filter No. 73 is a narrow-band filter while filters Nos. 58 and 25 transmit relatively broad regions of the spectrum. These filters are interposed between the primary light source and the target reticle, hence the spectral characteristics of the light emerging from the lens under test are determined by the filter used.

The foregoing procedure permits evaluation of longitudinal spherical aberration, $\Delta f$, on the axis of the lens. To make measurements of longitudinal spherical aberration for the tangential fan of a chief ray inclined at angle $\beta$ to the axis, it is only necessary to rotate the lens through the angle $\beta$ about the vertical axis of the nodal slide and to move the target away from the lens by amount $f$ (sec $\beta-1)$ which places the target in the focal plane at distance $f \tan \beta$ from the focal point. Measurements are then made in the same manner as was done for the axial region. A typical set of measurements is shown in table 2 for measurements made in the tangential fan for a chief ray inclined at angle $\beta=20^{\circ}$ to the axis of the lens. In this table, the column headed zone height $h$ gives the intervals along the bench scale at which observations were made. The value $h=0$ corresponds to the center of the emergent beam, plus values of $h$ indicate positions to one side, and negative values positions to the opposite side, of center. For each value of $h$, observed values of the angular deviation $\epsilon$ using each of three filters are listed under the filter Nos. 58,73 , and 25 ; the characteristics of these filters are given in table 1 . For positive values of $h$, negative values of $\epsilon$ indicate that the emergent beam is diverging or that the target is nearer to the lens than the focus. More generally when the sign of $\epsilon$ is the same as that of $h$, the emergent beam is converging; and when the sign of $\epsilon$ is the negative of the sign $h$, the emergent beam is diverging. The values of $\epsilon$ shown in table 2 indicate that the emergent beam is diverging and that the target is nearer to the lens than the focus for every zone. This is confirmed when the displacement $\Delta R_{T}$ of the target from the focal point is determined with the aid of
TABLE 2. Variation of parallax angle $\epsilon$ and apparent target displacement $\Delta R_{T}$ as a function of zone height $\mathrm{h}$ for a chief ray inclined $20^{\circ}$ with respect to the axis of the lens

These values were obtained for lens No, 1, a photographic objective having a focal length of $152.4 \mathrm{~mm}$. The values of $\epsilon$ were measured in the tangential fan for angle $\mathbf{B}=20^{\circ} \quad$ Values are given for three conditions of target illumination deter mined by Wratten filters Nos. 58,73 , and 25

\begin{tabular}{|c|c|c|c|c|c|c|}
\hline \multirow{2}{*}{$\begin{array}{c}\text { Zone } \\
\text { height } \\
h\end{array}$} & \multicolumn{3}{|c|}{$\begin{array}{c}\epsilon \\
\text { for filter Nos. }\end{array}$} & \multicolumn{3}{|c|}{$\begin{array}{c}\Delta R_{T} \\
\text { for filter Nos. }\end{array}$} \\
\hline & 58 & 73 & 25 & 58 & 73 & 25 \\
\hline $\begin{array}{c}m m \\
9 \\
8 \\
7 \\
6 \\
5 \\
4\end{array}$ & $\begin{array}{r}\text { sc. div. } \\
-34.5 \\
-29.8 \\
-23.7 \\
-17.1 \\
-11.9 \\
-7.9\end{array}$ & $\begin{array}{l}\text { sc. div. } \\
-28.1 \\
-24.5 \\
-19.6 \\
-13.9 \\
-9.5 \\
-5.9\end{array}$ & $\begin{array}{r}\text { s.div. } \\
-23.5 \\
-19.4 \\
-15.0 \\
-10.3 \\
-6.4 \\
-3.5\end{array}$ & $\begin{array}{l}\quad m m \\
-2.76 \\
-2.68 \\
-2.44 \\
-2.05 \\
-1.71 \\
-1.42\end{array}$ & $\begin{array}{l}\text { mm } \\
-2.25 \\
-2.20 \\
-2.01 \\
-1.66 \\
-1.37 \\
-1.06\end{array}$ & $\begin{aligned} & \mathrm{mm} \\
&-1.88 \\
&-1.74 \\
&-1.54 \\
&-1.23 \\
&-0.92 \\
&-.63\end{aligned}$ \\
\hline $\begin{array}{r}3 \\
2 \\
1 \\
0 \\
-1 \\
-2 \\
-3\end{array}$ & $\begin{array}{c}-5.0 \\
-2.6 \\
-1.2 \\
0 \\
1.4 \\
3.3 \\
5.0\end{array}$ & $\begin{array}{c}-3.3 \\
-1.3 \\
-1.1 \\
0 \\
0.5 \\
2.0 \\
2.9\end{array}$ & $\begin{array}{r}-1.5 \\
-0.5 \\
-.3 \\
0 \\
0.2 \\
.5 \\
1.2\end{array}$ & $\begin{array}{r}-1.20 \\
-0.93 \\
-.86 \\
-1.00 \\
-1.18 \\
-1.20\end{array}$ & $\begin{array}{r}-0.79 \\
-.46 \\
-.79 \\
-.76 \\
-.71 \\
-.70\end{array}$ & $\begin{array}{r}-.36 \\
-.18 \\
-.21 \\
--.14 \\
-.18 \\
-.29\end{array}$ \\
\hline $\begin{array}{l}-4 \\
-5 \\
-6 \\
-7 \\
-8 \\
-9\end{array}$ & $\begin{array}{r}8.1 \\
14.4 \\
20.5 \\
25.9 \\
30.8 \\
38.5\end{array}$ & $\begin{array}{r}5.7 \\
11.0 \\
16.5 \\
21.0 \\
26.1 \\
30.8\end{array}$ & $\begin{array}{r}3.1 \\
6.4 \\
12.1 \\
16.3 \\
20.0 \\
25.9\end{array}$ & $\begin{array}{l}-1.46 \\
-2.07 \\
-2.45 \\
-2.66 \\
-2.77 \\
-3.08\end{array}$ & $\begin{array}{l}-1.03 \\
-1.58 \\
-1.97 \\
-2.16 \\
-2.35 \\
-2.46\end{array}$ & $\begin{array}{r}-.56 \\
-.92 \\
-1.45 \\
-1.68 \\
-1.80 \\
-2.07\end{array}$ \\
\hline \multicolumn{4}{|c|}{ Average... } & -1.88 & -1.44 & -0.99 \\
\hline
\end{tabular}

eq (2). For these calculations, it is convenient to use the following modification of eq (2):

$$
\Delta R_{T}=\frac{k \epsilon f^{2} \sec ^{2} \beta}{h},
$$

where $k=27.35 \times 10^{-6}$ radians/scale division is used to convert $\epsilon$ from scale division to radians, $f$ is the focal length of the lens, and $\beta$ is the inclination of the chief ray to the lens axis. Values of $\Delta R_{T}$ derived from the observed values of $\epsilon$ listed in table 2 are shown in the same table under the heading $\Delta R_{T}$ for filters Nos. 58,73 , and 25 .

In table 2 , the values of $\Delta R_{T}$ for each value of $h$ and $\epsilon$ are negative as were indicated by opposing signs of $h$ and $\epsilon$. It is clear that the magnitude of $\Delta R_{T}$ varies appreciably with $h$. For comparison purposes, we may define the average of $\Delta R_{T}$ obtained with a given filter as the position of best focus with respect to the focal plane for use with that filter. In the present instance, it is noteworthy that the average value of $\Delta R_{T}$ is different for each filter and it is negative for each filter. Since the target was originally located in the plane of best axial focus, it is clear that for image-forming light incident upon the lens at an angular inclination of $\beta=20^{\circ}$ the plane of best focus for use with any one of these three filters is nearer to the lens than the plane of best axial focus.

This displacement of the plane of optimum focus in the extra-axial region from the plane of best axial focus arises from curvature of field and could not necessarily be anticipated at the start of the measurements. However, it does not invalidate the results, as variation of $\epsilon$ may arise from a combination of two effects (1) out-of-focus effect and (2) angular spherical 
aberration or parallax resulting from longitudinal spherical aberration inherent in the lens. These two effects are easily separable, as out-of-focus effect produces a variation of $\epsilon$ proportional to $h$ which expressed graphically plots in a straight line of constant slope passing through the zero point. In terms of $\Delta R_{T}$, it simply means the addition of a constant to all terms or displacing the entire longitudinal spherical aberration curve by a constant amount when $\Delta R_{T}$ is plotted as a function of $h$.

Examination of the values of $\Delta R_{T}$ for any one of the three sets of data shown in table 2 reveals an asymmetry in the manner in which $\Delta R_{T}$ varies with $h$ on opposite sides of the zero point of $h$. Thus in the column headed $73, \Delta R_{T}=-2.25 \mathrm{~mm}$ at $h=+9$ $\mathrm{mm}$ and $\Delta R_{T}=-2.46 \mathrm{~mm}$ at $h=-9 . \mathrm{mm}$. Differences of this nature may be ascribed to anyone of, or combination of, three causes which are (1) random error, (2) error in location of the center point, $h=0$; and (3) asymmetric aberrations in the lens. The effects of all three sources of error may be minimized by averaging the values of $\Delta R_{T}$ for paired values of $+h$ and $-h$ The results of such averaging of the values of $\Delta R_{T}$ given in table 2 are shown in table 3 . These values of $\Delta R_{T}$ shown in table 3 are therefore the accepted values of the longitudinal spherical aberration plus a constant which measures the departure of the position of best focus for rays in the tangential fan from the plane of best axial focus for a chief ray inclined at angle $\beta=20^{\circ}$ to the axis. As a check upon the accuracy of measurements, it is customary to repeat the entire series of measurements for the chief ray inclined at angle $\beta=-20^{\circ}$ to the axis. Since the lens is svmmetric about the optical axis, the values found for $\beta=-20^{\circ}$ are usually sufficiently nearly identical to the values found for $\beta=+20^{\circ}$ that the results may be averaged to produce the final accepted values of longitudinal spheri-
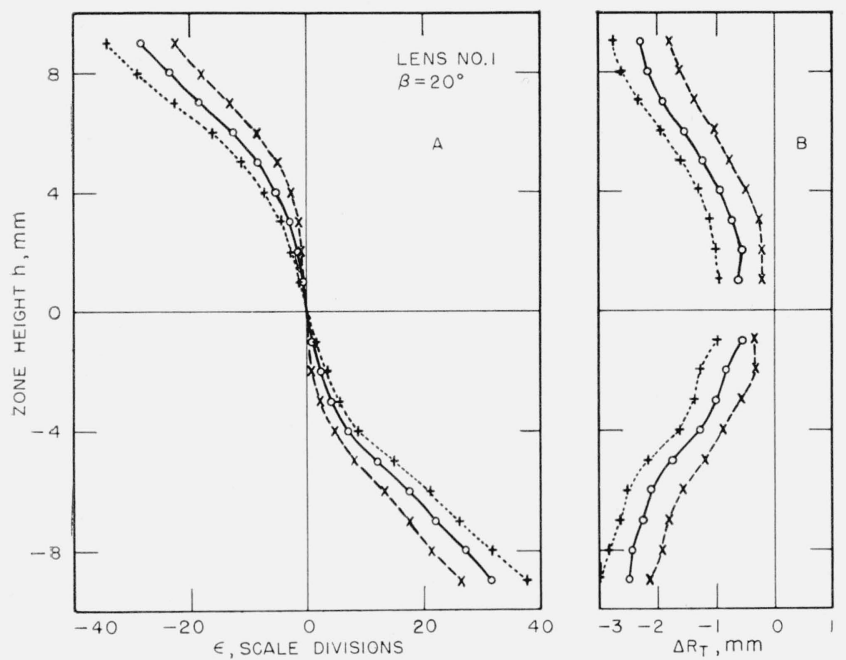

FIGURE 3. Variation of parallax angle, $\epsilon$, with zone height, $\mathrm{h}$, (frame $A$ ) and derived longitudinal spherical aterration, $\Delta \mathrm{R}_{\mathrm{T}}$, versus zone height $\mathrm{h}$ (frame $B$ ).

Results are given for a chief ray inclined at $\beta=20^{\circ}$ to the optical axis. In each of the irames, the dotted-line curves show results obtained with light transmitted by filter No 58; the solid-line curves show results for light transmitted by filter No. 73; and the dashed-line curves show results for light transmitted by filter No. 25 .
TABLE 3. Values of $\Delta \mathrm{R}_{T}$ and $\Delta\left(\Delta \mathrm{R}_{T}\right)$ as a funclion of zone height $\mathrm{h}$ for a chief ray inclined at $20^{\circ}$ to the axis of the lens

These results are for lens No. 1. The values of $\triangle R_{T}$ are derived from table 2 by averaging the values obtained at the same absolute value of zone height $h$. The values obtained with each of three filters (Wratten Nos, 58, 73, and 25) are shown. Negative values of $\Delta R_{T}$ indicate apparent displacement of the target shown. Negative values of $\Delta R_{T}$ indicate apparent displacement of the target
reticle along the chief ray and toward the lens with respect to the focal plane. Values of $\Delta\left(\Delta R_{T}\right)$ are given for the three filter pairs. All values are expressed in $\mathrm{mm}$.

\begin{tabular}{|c|c|c|c|c|c|c|}
\hline \multirow{2}{*}{ Zone height $h$} & \multicolumn{3}{|c|}{$\Delta R_{T}$ for filter No. } & \multicolumn{3}{|c|}{$\Delta\left(\Delta R_{T}\right)$ for filter pairs Nos. } \\
\hline & 58 & 73 & 25 & $73-58$ & $25-73$ & $25-58$ \\
\hline $\begin{array}{l}1 \\
2 \\
3 \\
3 \\
4 \\
5 \\
6 \\
7 \\
7 \\
8 \\
9\end{array}$ & $\begin{array}{l}-0.93 \\
-1.06 \\
-1.20 \\
-1.44 \\
-1.89 \\
-2.25 \\
-2.55 \\
-2.72 \\
-2.92\end{array}$ & $\begin{array}{l}-0.58 \\
-.58 \\
-.74 \\
-1.04 \\
-1.48 \\
-1.82 \\
-2.08 \\
-2.28 \\
-2.36\end{array}$ & $\begin{array}{l}-0.18 \\
-\quad .18 \\
-.32 \\
-.60 \\
-.92 \\
-1.34 \\
-1.61 \\
-1.77 \\
-1.98\end{array}$ & $\begin{array}{r}0.35 \\
.48 \\
.46 \\
.40 \\
.41 \\
.43 \\
.47 \\
.44 \\
.56\end{array}$ & $\begin{array}{r}0.40 \\
.40 \\
.42 \\
.44 \\
.56 \\
.48 \\
.47 \\
.51 \\
.38\end{array}$ & $\begin{array}{r}0.75 \\
.88 \\
.88 \\
.84 \\
.97 \\
.91 \\
.94 \\
.95 \\
.94\end{array}$ \\
\hline Average ........ & -1.88 & -1.44 & -0.99 & 0.44 & 0.45 & 0.89 \\
\hline$P E_{8-}$ & & & & \pm 0.04 & \pm 0.04 & \pm 0.05 \\
\hline
\end{tabular}

cal aberration for the chief ray inclined at $\beta=20^{\circ}$ to the axis. A graphical presentation showing both the averaged values of $\epsilon$ versus $h$ (frame $\mathrm{A}$ ) and $\Delta R_{T}$ versus $h$ (frame $\mathrm{B}$ ) for $\beta=+20^{\circ}$ and $\beta=-20^{\circ}$ is given in figure 3 . In this presentation, there was no averaging of values of $\epsilon$ and $\Delta R_{T}$ for paired values of $+h$ and $-h$, consequently the effect of small asymmetric aberrations are still present. In frame $B$ of figure 3, the effect of this asymmetric aberration is to tilt the whole array of $\Delta R_{T}$ curves slightly in a clockwise manner. In the final determination, average values of $\Delta R_{T}$ are obtained from paired values of $+\beta$ and $-\beta$, and for paired values of $+h$ and $-h$.

\subsection{In the Sagittal Fan}

To make measurements of longitudinal spherical aberration for the sagittal fan of a chief ray inclined at angle $\beta$ to the axis, it is necessary to rotate the lens about a horizontal axis normal to the optical axis and passing through the rear nodal point of the lens and to move the target away from the lens by an amount $f$ (sec $\beta-1)$. This places the target at distance $f$ sec $\beta$ from the rear nodal point of the lens as measured along the chief ray inclined at angle $\beta$ to the axis and at distance $f$ tan $\beta$ from the axial focal point as measured in the focal plane. The arrangement of apparatus is shown in figure 4 . Measurements of $\epsilon$ versus $h$ are then made in the same manner as for the axial region. In order that the measurements are made for the same region of the picture area as those made in the tangential fan, the lens is also rotated $90^{\circ}$ about its own optical axis from the position occupied during measurements in the tangential fan. Except for the foregoing differences, the process of measurement is essentially the same as that employed for the tangential fan. Values of the displacement $\Delta R_{S}$ of the target from the focal point of the lens for the given chief ray are obtained with the aid of eq (4). The final accepted values of $\Delta R_{s}$ are obtained from the 


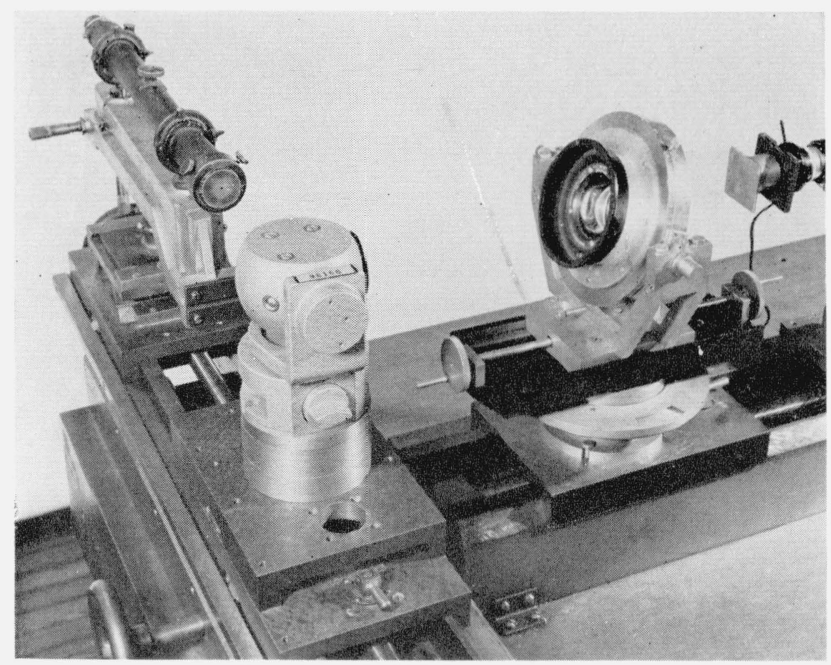

Figure 4. Test equipment arranged for measurement in the sagittal fan.

The same equipment appearing in figure 2 is shown with the lens rotated through an angle $\beta$ about the horizontal axis.

average of paired values for $+\beta$ and $-\beta$ and for paired values of $+h$ and $-h$.

\section{Results of Measurement}

The results of measurement on three wide-angle lenses having focal lengths of approximately 150 $\mathrm{mm}$ are presented in this study. Two of the lenses, designated Nos. 1 and 2, are essentially distortionfree while the third lens, designated No. 3, has a moderate amount of distortion.

\subsection{Longitudinal Spherical Aberration}

The values of the longitudinal spherical aberration for both the tangential and sagittal fans are shown graphically in figures 5, 6, and 7. In the upper series of frames of each figure, average values of the displacement $\Delta R_{T}$ for the tangential fan are given as a function of zone height $h$ at angular inclinations of the chief ray from the axis $0,10,20,30$, and 40 degrees. Results for the same series of filters are presented for each lens for each angular inclination. In each of the figures, the dotted-line curves show the results obtained with light transmitted by filter No. 58 , the solid line curves show the results obtained for light transmitted by filter No. 73; and the dashed line curves show the results obtained for light transmitted by filter No. 25. The optical characteristics of these filters are given in table 1 . In the lower series of frames in figures 5,6 , and 7 values of the displacement $\Delta R_{s}$ for the sagittal fan as a function of zone height $h$, are given for the same angular inclinations of the chief ray to the optical axis and for light transmitted by the same three filters. In the graphs, values of $\Delta R_{s}$ and $\Delta R_{s}$ are plotted for both positive and negative values of $h$. This was done to increase clarity for, although the lower portion of a given curve is a mirror image of the upper portion, the inclusion of both upper and lower portion permits a better visualization of the phenomenon. In contemplating these curves, it is interesting to note the variation in shape with increasing $\beta$. In all cases shown, the curves of $\Delta R_{T}$ versus $h$ become increasingly concave toward the axis and the curves of $\Delta R_{s}$ versus $h$ become increasingly convex with respect to the axis with increasing values of $\beta$.

Too, it is evident that the zero points of $\Delta R_{T}$ and $\Delta R_{s}$ do not always maintain the same relative position with respect to the curves of $\Delta R_{T}$ and $\Delta R_{s}$ for various values of $\beta$. This shift results from curvature of field and the magnitude of the shift is a measure of tangential field curvature in the case of the $\Delta R_{T}$ values of the sagittal field curvature in the case of $\Delta R_{s}$ values. It mav also be noted that the range of values of $h$ decreases with $\beta$ in the tangential fan but remains constant for the sagittal fan. This reduction in range is caused by vignetting which has a more pronounced effect in the tangential fan.

\subsection{Longitudinal Chromatic Aberration}

In figures 5, 6, and 7, it is clear that the longitudinal spherical aberration curves obtained with the various filters for a given value of $\beta$ are very similar in appearance but are displaced along the chief ray with respect to one another. This displacement of the curves with respect to one another may be ascribed to longitudinal chromatic aberration as the effective wavelength of the light illuminating the target reticle varies from one filter to another as may be inferred from the information contained in table 1. Hence it is reasonable to suppose that the magnitude of the displacement of one curve with respect to another may be taken as a measure of the change in focal position along the chief ray produced by the change in character of the light transmitted by one filter as compared to another. While strictly speaking the term "longitudinal chromatic aberration" applies only to differences in focal position induced by change in the nature of the image forming light from one monochromatic illuminant having a sharply defined wavelength to another monochromatic illuminant having a different sharply defined wavelength, it is nonetheless at times expedient to use the term when dealing with image forming light whose nature is determined by a filter. Hence in this study the term "longitudinal chromatic aberration" will be used as a convenient mnemonic to designate the change in focal position produced by a change in the filter controlling the natures of the illuminant. With this as a basis, the longitudinal chromatic aberration in the tangential fan for a given filter pair may be determined zone-by-zone from the relation

$$
\Delta\left(\Delta R_{T}\right)=\Delta R_{T}-\Delta R_{T}^{\prime}
$$

where $\Delta R_{T}$ is the longitudinal spherical aberration in the tangential fan determined for a specified value of $h$ when using a given light filter and $\Delta R^{\prime}{ }_{T}$ is that determined for the same value of $h$ when using 

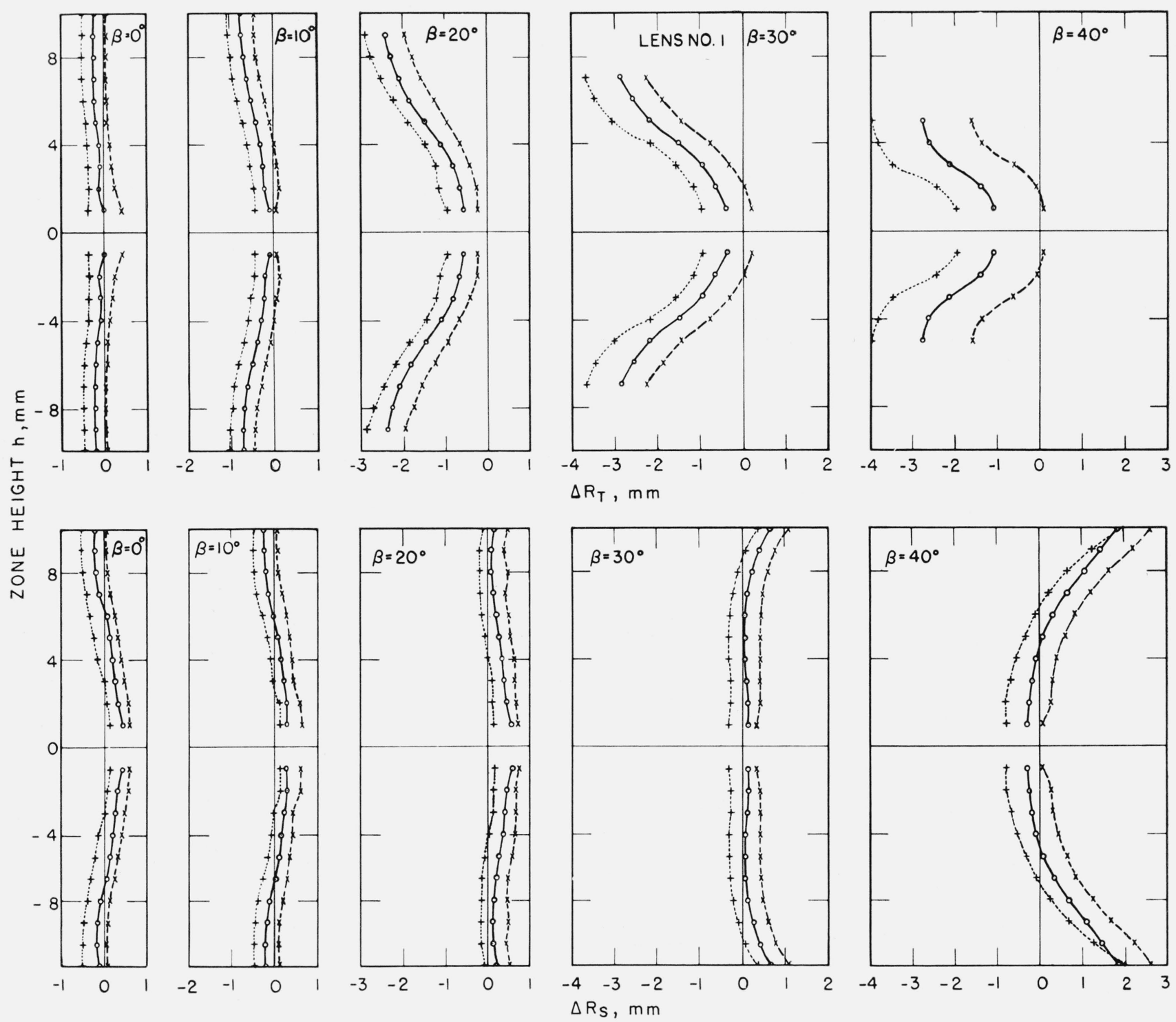

FIGURE 5. Variation of longitudinal spherical aberration, $\Delta \mathrm{R}_{\mathrm{T}}$, in the langential fan and, $\Delta \mathrm{R}_{\mathrm{s}}$, in the sagittal fan with zone height, $\mathrm{h}$, for five values of $\beta$.

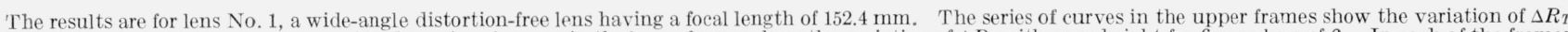

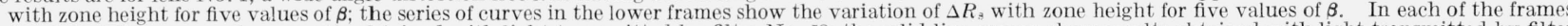

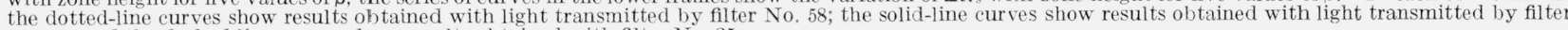
No. 73; and the dashed-line curves show results obtained with filter No. 25. 


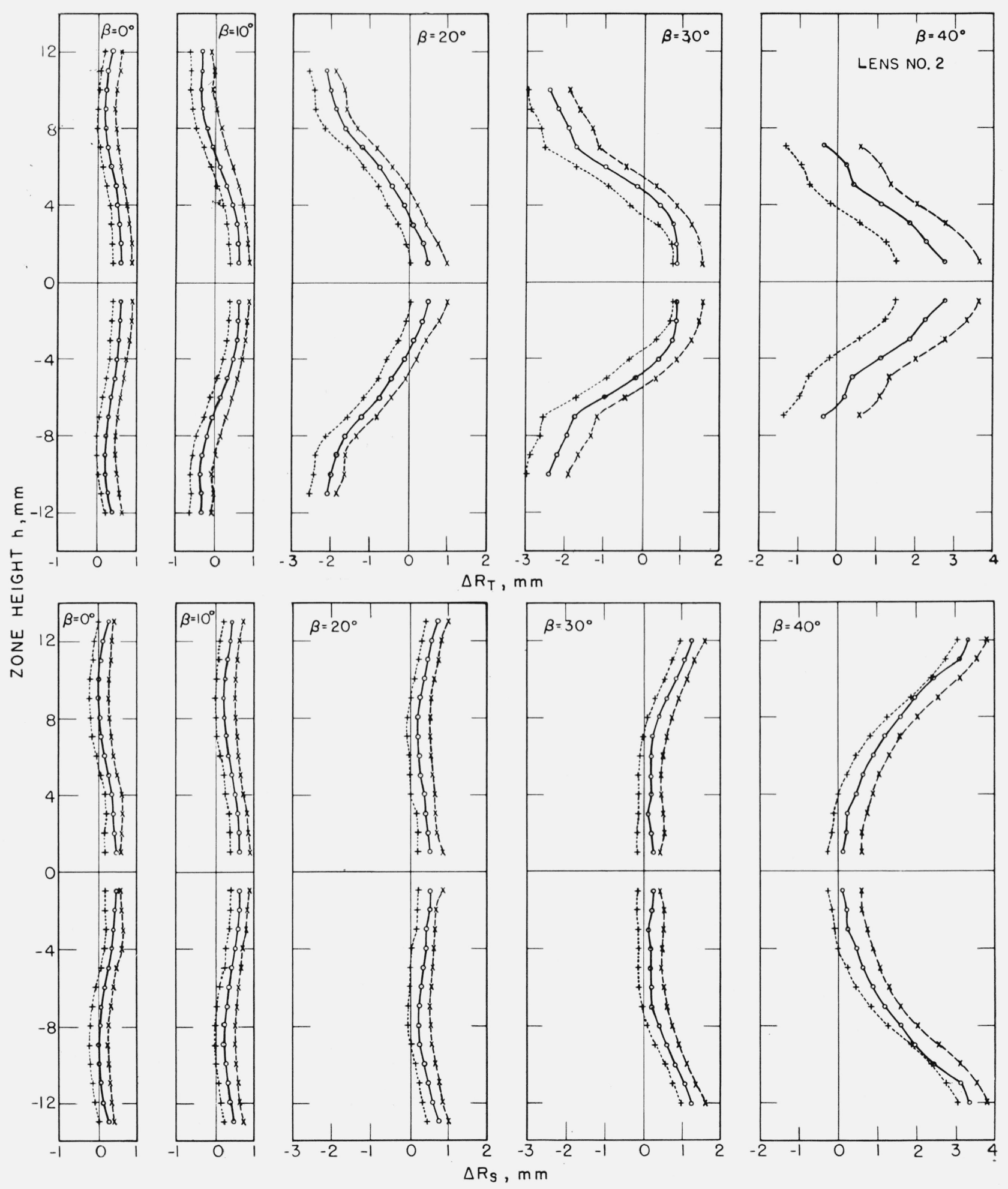

FIGURE 6. Variation of longitudinal spherical aberration, $\Delta \mathrm{R}_{\mathrm{T}}$, in the tangential fan and, $\Delta \mathrm{R}_{\mathrm{s}}$, in the sagittal fan with zone height, $\mathrm{h}$, for five values of $\beta$.

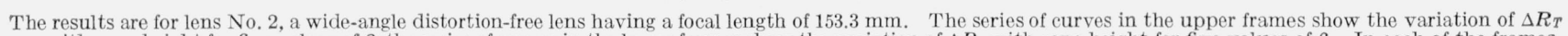

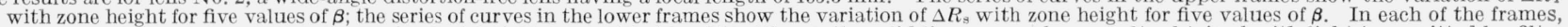

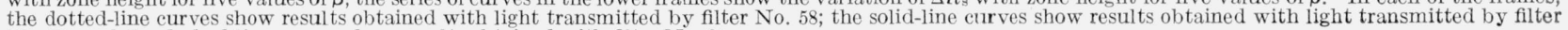
No. 73; and the dashed-line curves show results obtained with filter No. 25. 

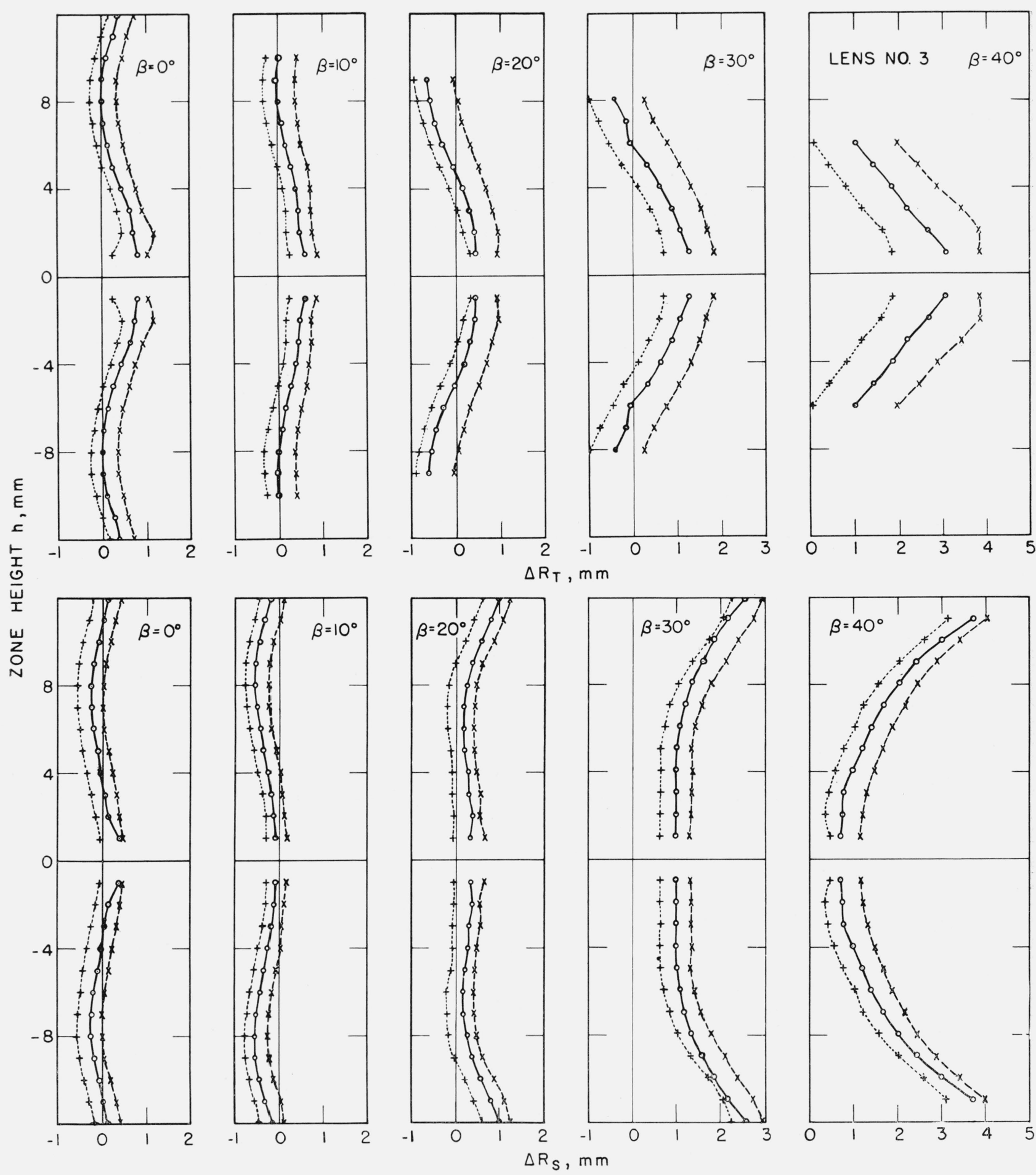

FIGURE 7. Variation of longitudinal spherical aberration, $\Delta \mathrm{R}_{\mathrm{T}}$, in the tangential fan and, $\Delta \mathrm{R}_{\mathrm{s}}$, in the sagittal fan with zone height, $\mathrm{h}$, for five values of $\beta$.

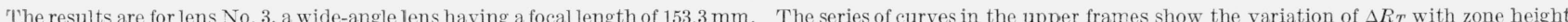

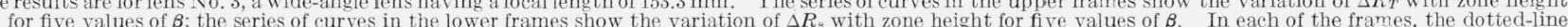

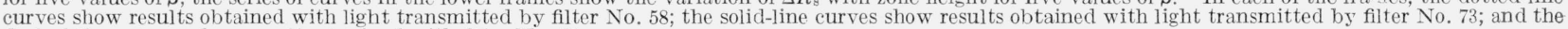
dashed-line curves show results obtained with filter No. 25. 
a different light filter. In a similar manner, the relation

$$
\Delta\left(\Delta R_{s}\right)=\Delta R_{s}-\Delta R_{s}^{\prime}
$$

may be used to determine the longitudinal chromatic aberration in the sagittal fan. It is clear from figures 5,6 , and 7 that for a given value of $\beta$, the quantities $\Delta\left(\Delta R_{T}\right)$ and $\Delta\left(\Delta R_{s}\right)$ do not vary appreciably with zone height for these three lenses.

The invariance of $\Delta\left(\Delta R_{T}\right)$ with zone height is also indicated in table 3 where values of $\Delta\left(\Delta R_{T}\right)$ are given for three filter pairs. The average values of $\overline{\Delta\left(\Delta R_{T}\right)}$ are also given together with the probable error of a single observation $\left(P E_{s}\right)$. In view of the various errors inherent in the measurement of $\Delta R_{T}$, it is reasonable to conclude from the low values of $P E_{s}$ that the quantity $\Delta\left(\Delta R_{T}\right)$ may be regarded as invariant for these lenses. This leads to a simplification in the determination of $\overline{\Delta\left(\Delta R_{T}\right)}$ as one may determine the average value of $\Delta R_{T}$ for each filter used and evaluate $\overline{\Delta\left(\Delta R_{T}\right)}$ for any pair of filters from the relation

$$
\overline{\Delta\left(\Delta R_{T}\right.}=\overline{\Delta R_{T}}-\overline{\Delta R_{T}^{\prime}} .
$$

In a similar manner, the average value of $\overline{\Delta\left(\Delta R_{s}\right)}$ for the sagittal fan for a given value of $\beta$ may be determined from the relation

$$
\overline{\Delta\left(\Delta R_{s}\right)}=\overline{\Delta R_{s}}-\overline{\Delta R_{s}^{\prime}} .
$$

TABLE 4. Values of $\overline{\Delta \mathrm{R}_{\mathrm{T}}}, \overline{\Delta \mathrm{R}_{\mathrm{s}}}, \overline{\Delta\left(\Delta \mathrm{R}_{\mathrm{T}}\right)}$, and $\overline{\Delta\left(\Delta \mathrm{R}_{\mathrm{s}}\right)}$ for the tangential and sagittal fans as a function of $\beta$

These values are for lens No. 1 a wide-angle distortion-free lens having a focal length of $152.4 \mathrm{~mm}$. Part (a) shows the values of $\overline{\Delta\left(R_{T}\right)}$ and ' $\overline{\Delta\left(\Delta R_{T}\right)}$ for the tangential fan and part (b) shows the corresponding values for the sagittal fan. Values are given for three conditions of target illumination determined by the indicated

\begin{tabular}{|c|c|c|c|c|c|c|}
\hline \multirow{2}{*}{$\begin{array}{c}\beta \\
\text { degrees }\end{array}$} & \multicolumn{3}{|c|}{$\begin{array}{l}\overline{\Delta R_{T}} \\
\text { for filter No. }\end{array}$} & \multicolumn{3}{|c|}{$\begin{array}{l}\qquad \overline{\Delta\left(\Delta R_{T}\right)} \\
\text { for filter pairs Nos. }\end{array}$} \\
\hline & 58 & 73 & 25 & $73-58$ & $25-73$ & $25-58$ \\
\hline $\begin{array}{r}0 \\
5 \\
10 \\
15 \\
20 \\
25 \\
30 \\
35 \\
40 \\
45\end{array}$ & $\begin{array}{r}-0.41 \\
-.46 \\
-.76 \\
-1.27 \\
-1.88 \\
-2.21 \\
-2.26 \\
-2.44 \\
-3.12 \\
-5.76\end{array}$ & $\begin{array}{r}-0.15 \\
-.17 \\
-.45 \\
-.90 \\
-1.47 \\
-1.64 \\
-1.58 \\
-1.53 \\
-2.01 \\
-4.42\end{array}$ & $\begin{array}{r}0.14 \\
.08 \\
-.16 \\
-. .57 \\
-1.01 \\
-1.22 \\
-0.90 \\
-.73 \\
-. .72 \\
-2.84\end{array}$ & $\begin{array}{r}0.26 \\
.29 \\
.31 \\
.37 \\
.41 \\
.57 \\
.68 \\
.91 \\
1.11 \\
1.34\end{array}$ & $\begin{array}{r}0.29 \\
.25 \\
.29 \\
.33 \\
.46 \\
.42 \\
.68 \\
.80 \\
1.29 \\
1.58\end{array}$ & $\begin{array}{r}0.55 \\
.54 \\
.60 \\
.70 \\
.87 \\
.99 \\
1.36 \\
1.71 \\
2.40 \\
2.92\end{array}$ \\
\hline
\end{tabular}
filter numbers. All values are expressed in $\mathrm{mm}$.

(a) Tangential fan

\begin{tabular}{|c|c|c|c|c|c|c|}
\hline \multirow{2}{*}{$\begin{array}{c}\beta \\
\text { degrees }\end{array}$} & \multicolumn{3}{|c|}{ for filter No. } & \multicolumn{3}{|c|}{$\begin{array}{l}\frac{\overline{\Delta\left(\Delta R_{s}\right)}}{\text { for filter pairs Nos. }} \\
\text { Noir }\end{array}$} \\
\hline & 58 & 73 & 25 & $73-58$ & $25-73$ & $25-58$ \\
\hline $\begin{array}{r}0 \\
5 \\
10 \\
15 \\
20 \\
25 \\
30 \\
35 \\
40 \\
45\end{array}$ & $\begin{array}{r}-0.24 \\
.02 \\
-.20 \\
-.04 \\
-.02 \\
-.30 \\
-.18 \\
-.08 \\
-.09 \\
-.04\end{array}$ & $\begin{array}{r}0.08 \\
.28 \\
.04 \\
.24 \\
.27 \\
.02 \\
.19 \\
.32 \\
.47 \\
.54\end{array}$ & $\begin{array}{r}0.30 \\
.52 \\
.32 \\
.48 \\
.54 \\
.32 \\
.52 \\
.71 \\
1.02 \\
1.02\end{array}$ & $\begin{array}{r}0.32 \\
.26 \\
.24 \\
.28 \\
.29 \\
.32 \\
.37 \\
.40 \\
.38 \\
.58\end{array}$ & $\begin{array}{r}0.22 \\
.24 \\
.28 \\
.24 \\
.27 \\
.30 \\
.33 \\
.39 \\
.55 \\
.48\end{array}$ & $\begin{array}{r}0.54 \\
.50 \\
.52 \\
.52 \\
.56 \\
.62 \\
.70 \\
.79 \\
.93 \\
1.66\end{array}$ \\
\hline
\end{tabular}

(b) Sagittal fan
Further consideration of the curves in figures 5, 6, and 7 indicates that both $\overline{\Delta\left(\Delta R_{T}\right)}$ and $\overline{\Delta\left(\Delta R_{s}\right)}$ increase with increasing $\beta$ and that for a given value of $\beta$ the value of $\overline{\Delta\left(\Delta R_{T}\right)}$ is greater than that of $\overline{\Delta\left(\Delta R_{s}\right)}$. The variation of $\overline{\Delta\left(\Delta R_{T}\right)}$ and $\overline{\Delta\left(\Delta R_{s}\right)}$ with $\beta$ is shown in tables 4,5 , and 6 for three filter pairs for lenses Nos. 1, 2, and 3 . In each table, the values of $\overline{\Delta R_{T}}$ and $\overrightarrow{\Delta R_{s}}$ obtained for light trans-

TABLE 5. Values of $\overline{\Delta \mathrm{R}_{\mathrm{T}}}, \overline{\Delta \mathrm{R}_{\mathrm{s}}}, \overline{\Delta\left(\Delta \mathrm{R}_{\mathrm{T}}\right)}$, and $\overline{\Delta\left(\Delta \mathrm{R}_{\mathrm{s}}\right)}$ for the tangential and sagittal fans as a function of $\beta$.

These values are for lens No. 2, a wide-angle distortion-free lens having a focal length of $153.3 \mathrm{~mm}$. Part (a) shows the values of $\overline{\Delta R_{T}}$ and $\overline{\Delta\left(\Delta R_{T}\right)}$ for the tangential fan and part (b) shows the corresponding values for the sagittal fan. Values are given for three conditions of target illumination determined by the

\begin{tabular}{|c|c|c|c|c|c|c|}
\hline \multirow{2}{*}{$\begin{array}{c}\beta \\
\text { degrees }\end{array}$} & \multicolumn{3}{|c|}{$\begin{array}{l}\overline{\Delta R_{T}} \\
\text { for filter No. }\end{array}$} & \multicolumn{3}{|c|}{$\begin{array}{c}\overline{\frac{\Delta(\Delta R T)}{}} \\
\text { for filter pairs Nos. }\end{array}$} \\
\hline & 58 & 73 & 25 & 73-58 & $25-73$ & $25-58$ \\
\hline $\begin{array}{c}0 \\
10 \\
20 \\
30 \\
40\end{array}$ & $\begin{array}{r}0.19 \\
-.15 \\
-1.25 \\
-1.20 \\
.00\end{array}$ & $\begin{array}{r}0.39 \\
.10 \\
-.82 \\
-.65 \\
1.16\end{array}$ & $\begin{array}{r}0.65 \\
.39 \\
-.48 \\
-.10 \\
2.11\end{array}$ & $\begin{array}{r}0.20 \\
.25 \\
.43 \\
.55 \\
1.16\end{array}$ & $\begin{array}{r}0.26 \\
.29 \\
.34 \\
.55 \\
.95\end{array}$ & $\begin{array}{r}0.46 \\
.54 \\
.77 \\
1.10 \\
2.11\end{array}$ \\
\hline
\end{tabular}
indicated filter Nos. All values are expressed in $\mathrm{mm}$.

(a) Tangential fan

\begin{tabular}{|c|c|c|c|c|c|c|}
\hline \multirow{2}{*}{$\begin{array}{c}\boldsymbol{\beta} \\
\text { degrees }\end{array}$} & \multicolumn{3}{|c|}{$\begin{array}{c}\overline{\Delta R_{s}} \\
\text { for filter No. }\end{array}$} & \multicolumn{3}{|c|}{$\begin{array}{c}\overline{\Delta\left(\Delta R_{s}\right)} \\
\text { for filter pairs Nos. }\end{array}$} \\
\hline & 58 & 73 & 25 & $73-58$ & $25-73$ & $25-58$ \\
\hline $\begin{array}{r}0 \\
10 \\
20 \\
30 \\
40\end{array}$ & $\begin{array}{r}-0.01 \\
.18 \\
.15 \\
.14 \\
.81\end{array}$ & $\begin{array}{r}0.21 \\
.41 \\
.43 \\
.44 \\
1.17\end{array}$ & $\begin{array}{r}0.44 \\
.66 \\
.70 \\
.77 \\
1.62\end{array}$ & $\begin{array}{r}0.22 \\
.23 \\
.28 \\
.30 \\
.36\end{array}$ & $\begin{array}{r}0.23 \\
.25 \\
.27 \\
.33 \\
.45\end{array}$ & $\begin{array}{r}0.45 \\
.48 \\
.55 \\
.63 \\
.81\end{array}$ \\
\hline
\end{tabular}

(b) Sagittal fan

TABLE 6. Values of $\overline{\Delta \mathrm{R}_{\mathrm{T}}}, \overline{\Delta \mathrm{R}_{\mathrm{s}}}, \overline{\Delta\left(\Delta \mathrm{R}_{\mathrm{T}}\right)}$, and $\overline{\Delta\left(\Delta \mathrm{R}_{\mathrm{s}}\right)}$ for the tangential and sagittal fans as a function of $\beta$.

The values are for lens No. 3, a wide-angle lens having a focal length of 153.3 mm. Part (a) shows the values of $\overline{\Delta R_{T}}$ and $\overline{\Delta\left(\Delta R_{T}\right)}$ for the tangential fan and

\begin{tabular}{|c|c|c|c|c|c|c|}
\hline \multirow{2}{*}{$\begin{array}{c}\boldsymbol{\beta} \\
\text { degrees }\end{array}$} & \multicolumn{3}{|c|}{$\begin{array}{l}\overline{\Delta R_{T}} \\
\text { for filter No. }\end{array}$} & \multicolumn{3}{|c|}{$\begin{array}{l}\qquad \overline{\Delta\left(\Delta R_{T}\right)} \\
\text { for filter pairs Nos. }\end{array}$} \\
\hline & 58 & 73 & 25 & $73-58$ & $25-73$ & $25-58$ \\
\hline $\begin{array}{r}0 \\
10 \\
20 \\
30 \\
40\end{array}$ & $\begin{array}{r}0.05 \\
-.07 \\
-.33 \\
-.10 \\
.99\end{array}$ & $\begin{array}{r}0.31 \\
. .22 \\
-.08 \\
.44 \\
2.04\end{array}$ & $\begin{array}{r}0.67 \\
.56 \\
.49 \\
1.12 \\
3.06\end{array}$ & $\begin{array}{r}0.26 \\
.29 \\
.25 \\
.54 \\
1.05\end{array}$ & $\begin{array}{r}0.36 \\
.34 \\
.57 \\
.68 \\
1.02\end{array}$ & $\begin{array}{r}0.62 \\
.63 \\
.82 \\
1.22 \\
2.07\end{array}$ \\
\hline
\end{tabular}
part (b) shows the corresponding values for the sagittal fan. V Vlues are given
for three conditions of target illumination determined by the indicated filter for three conditions of target illumin.
Nos. All values are expressed in $\mathrm{mm}$.

\begin{tabular}{|c|c|c|c|c|c|c|}
\hline \multirow{2}{*}{$\begin{array}{c}\beta \\
\text { degrees }\end{array}$} & \multicolumn{3}{|c|}{ for filter No. } & \multicolumn{3}{|c|}{$\begin{array}{l}\overline{\Delta\left(\Delta R_{s}\right)} \\
\text { for filter pairs Nos. }\end{array}$} \\
\hline & 58 & 73 & 25 & $73-58$ & $25-73$ & $25-58$ \\
\hline $\begin{array}{r}0 \\
10 \\
20 \\
30 \\
40\end{array}$ & $\begin{array}{r}-0.35 \\
.45 \\
.02 \\
1.10 \\
1.30\end{array}$ & $\begin{array}{r}-0.03 \\
.67 \\
.41 \\
1.40 \\
1.70\end{array}$ & $\begin{array}{r}0.23 \\
.96 \\
.65 \\
1.81 \\
2.14\end{array}$ & $\begin{array}{r}0.32 \\
.22 \\
.39 \\
.30 \\
.40\end{array}$ & $\begin{array}{r}0.26 \\
.29 \\
.24 \\
.41 \\
.44\end{array}$ & $\begin{array}{r}0.58 \\
.51 \\
.63 \\
.71 \\
.84\end{array}$ \\
\hline
\end{tabular}

(b) Sagittal fan 

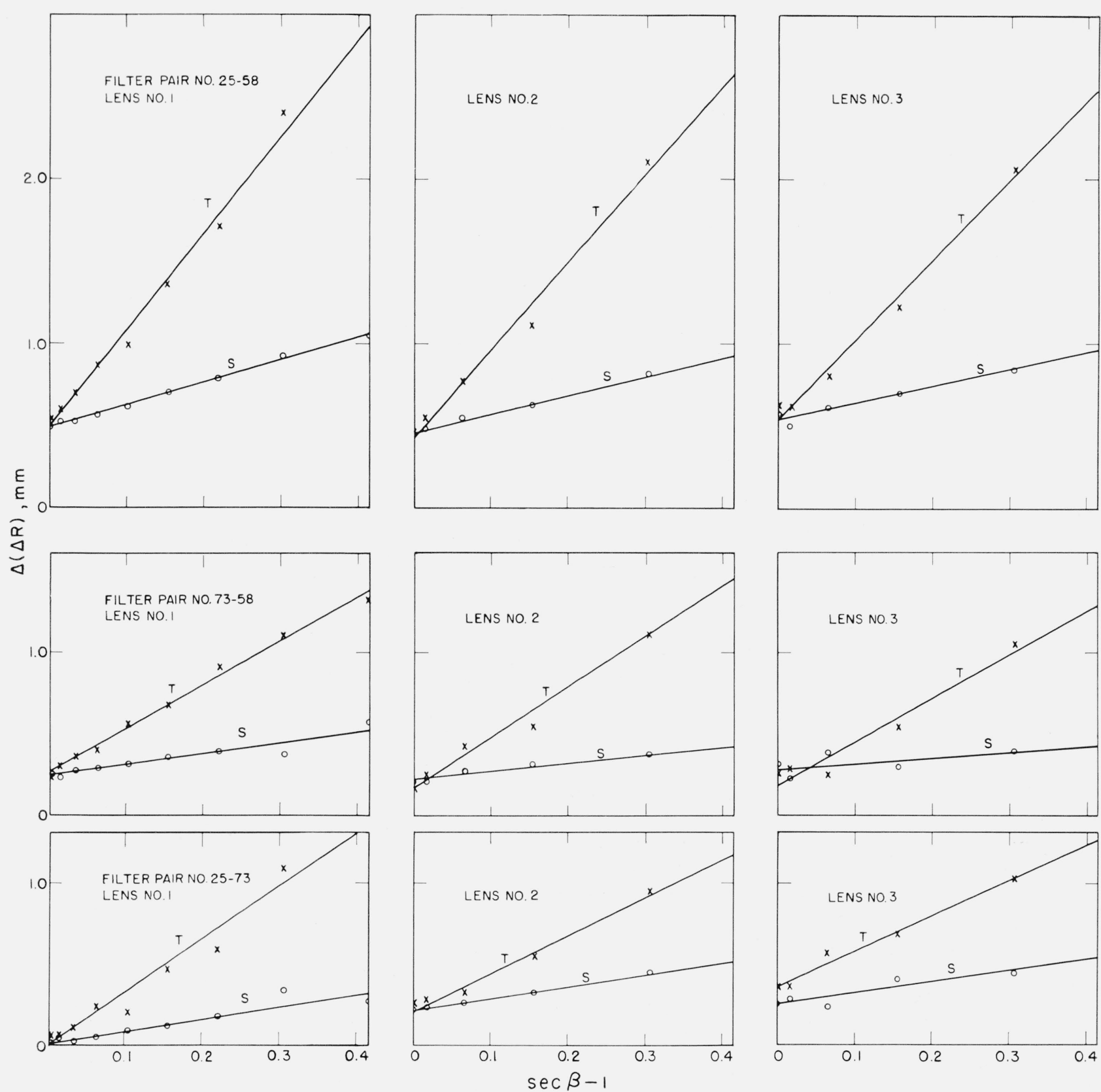

FIgURE 8. $\overline{\Delta(\Delta \mathrm{R})}$ versus ( $\sec \beta-1)$ curves for three lenses for three filter pairs.

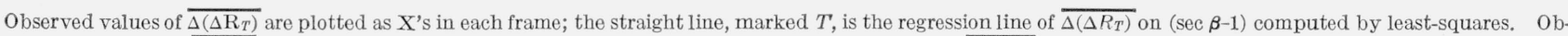

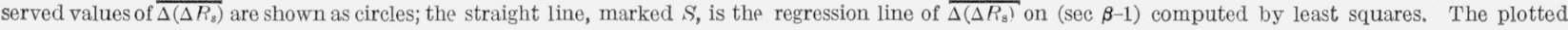
values are taken from table 5 .

mitted by each filter for each value of $\beta$ are also given. The values of $\overline{\Delta\left(\Delta R_{T}\right)}$ and $\overline{\Delta\left(\Delta R_{s}\right)}$ for each filter pair are derived from these values of $\overline{\Delta R_{T}}$ and $\overline{\Delta R_{s}}$ with the aid of eqs (7) and (8).

When the values of $\overline{\Delta\left(\Delta R_{T}\right)}$ and $\overline{\Delta\left(\Delta R_{s}\right)}$ are plotted as a function of $(\sec \beta-1)$ as shown in figure 8 , it found that the variation of these quantities with $(\sec \beta-1)$ is close to linear. Accordingly one may write an empirical formula connecting $\overline{\Delta\left(\Delta R_{T}\right)}$ with $(\sec \beta-1)$ as follows:

$$
\overline{\Delta\left(\Delta R_{T}\right)}=a+b(\sec \beta-1)
$$

which is the equation of a straight line with $a$ as the $y$-intercept and $b$ as the slope of the line. A similar empirical formula of the same nature may be written connecting $\overline{\Delta\left(\Delta R_{s}\right)}$ and (sec $\left.\beta-1\right)$. The constants $a$ and $b$ may be determined graphically by drawing the best fitting straight line or analytically by 
TABLE 7. Values of the constants a and $\mathrm{b}$ for fitting an equation of the form $\overline{\Delta(\Delta \mathrm{R})}=\mathrm{a}+\mathrm{b}(\sec \beta-1)$ to the observed values of $\overline{\Delta\left(\mathrm{R}_{\mathrm{T}}\right)}$ and $\overline{\Delta\left(\Delta \mathrm{R}_{\mathrm{s}}\right)}$ as a function of $(\sec \beta-1)$.

Values of $a$ and $b$ are given for three filter pairs for both $\overline{\Delta\left(\Delta R_{T}\right)}$ and $\overline{\Delta\left(\Delta R_{s}\right)}$ for each of three lenses. All values are expressed in $\mathrm{mm}$.

\begin{tabular}{|c|c|c|c|c|c|}
\hline \multirow{3}{*}{$\begin{array}{l}\text { Lens } \\
\text { No. }\end{array}$} & \multirow{3}{*}{$\begin{array}{l}\text { Filter } \\
\text { pairs } \\
\text { Nos. }\end{array}$} & \multicolumn{4}{|c|}{ 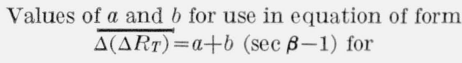 } \\
\hline & & \multicolumn{2}{|c|}{$\overline{\Delta\left(\Delta R_{T}\right)}$} & \multicolumn{2}{|c|}{$\overline{\Delta\left(\Delta R_{s}\right)}$} \\
\hline & & $a$ & $b$ & $a$ & $b$ \\
\hline 1 & $\begin{array}{l}73-58 \\
25-73 \\
25-58\end{array}$ & $\begin{array}{r}0.27 \\
.22 \\
.49\end{array}$ & $\begin{array}{l}\text { 2. } 67 \\
\text { 3. } 21 \\
5.88\end{array}$ & $\begin{array}{r}0.26 \\
.23 \\
.49\end{array}$ & $\begin{array}{r}0.64 \\
.73 \\
1.37\end{array}$ \\
\hline 2 & $\begin{array}{l}73-58 \\
25-73 \\
25-58\end{array}$ & $\begin{array}{r}0.19 \\
.23 \\
.43\end{array}$ & $\begin{array}{l}3.02 \\
2.27 \\
5.28\end{array}$ & $\begin{array}{r}0.23 \\
.23 \\
.46\end{array}$ & $\begin{array}{r}0.47 \\
.71 \\
1.17\end{array}$ \\
\hline 3 & $\begin{array}{l}73-58 \\
25-73 \\
25-58\end{array}$ & $\begin{array}{r}0.19 \\
.36 \\
.55\end{array}$ & $\begin{array}{l}2.63 \\
2.18 \\
4.82\end{array}$ & $\begin{array}{l}0.29 \\
.26 \\
.55\end{array}$ & $\begin{array}{r}0.33 \\
.67 \\
1.01\end{array}$ \\
\hline
\end{tabular}

applying the method of least squares. It is evident from the curves shown in figure 8 that the constant $a$ is approximately equal to the value of $\overline{\Delta\left(\Delta R_{T}\right)}$ or $\overline{\Delta\left(\Delta R_{s}\right)}$ at $\beta=0^{\circ}$. It is also evident that the increase in $\overline{\Delta\left(\Delta R_{T}\right)}$ with $(\sec \beta-1)$ is substantially greater than that of $\overline{\Delta\left(\Delta R_{s}\right)}$; accordingly the slope $b$ is greater for the tangential fan than for the sagittal fan.

Determinations of the constants $a$ and $b$ that are usable in equations of the form given in eq (9) for showing the relation of values of $\overline{\Delta\left(\Delta R_{T}\right)}$ or $\overline{\Delta\left(\Delta R_{s}\right)}$ to (sec $\beta-1$ ) were made for each of the filter pairs for each of the three lenses using the values given in tables 4,5 , and 6 . The values of $a$ and $b$ for each combination are given in table 7 . The straight line shown in each of the frames in figure 8 is drawn to satisfy the equation of the line fixed by the least squares determination of values of $a$ and $b$ for the particular filter pair. It is clear from the manner in which the points in any one of the graphs are distributed with respect to the straight line in that graph that the assumption of a linear relationship between values of $\overline{\Delta\left(\Delta R_{T}\right)}$ and $(\sec \beta-1)$ and between values of $\overline{\Delta\left(\Delta R_{s}\right)}$ and $(\sec \beta-1)$ is a valid one.

For a given filter pair, it is clear that the value of the slope $b$ found for the $\overline{\Delta\left(\Delta R_{T}\right)}$ data is not the same as that for the $\overline{\Delta\left(\Delta R_{s}\right)}$ data. The value of the slope $b$ for the $\overline{\Delta\left(\Delta R_{T}\right)}$ data is in all instances greater by a ratio ranging from 3.2 to 7.9 times greater than that for the $\overline{\Delta\left(\Delta R_{s}\right)}$ data. In view of the relative magnitude of this ratio, it is believed that it may be regarded as established that the variation of $\overline{\Delta\left(\Delta R_{T}\right)}$ with $(\sec \beta-1)$ is significantly greater than that of $\overline{\Delta\left(\Delta R_{s}\right)}$ for the three lenses.

\section{Discussion}

In this study, a visual method of determining longitudinal spherical and chromatic aberration from measurements of angular deviation in the collimated beam emergent from a lens has been extended to the extra-axial region. Results are reported for measurements made on three lenses at five angular inclinations to the optical axis. It is of especial interest to note the increase in the values of longitudinal spherical aberration with increasing $\beta$ and to note the large magnitudes attained. At $\beta=40^{\circ}$, a range of $3 \mathrm{~mm}$ in the measured values of the longitudinal spherical aberration is not unusual; of course if these values were referred to the normal to the focal plane they would be reduced by a cosine factor but would still remain quite large.

The authors express their appreciation to other members of the staff of the National Bureau of Standards for assistance during this work and in particular to Edgar C. Watts who prepared the illustrations.

(Paper 66C3-95) 